\title{
Reformas de New Public Management: el caso de la Administración sanitaria catalana
}

Raquel Gallego Calderón *

\section{New Public Management y Reforma Sanitaria: un marco teórico}

La reforma de la Administración sanitaria catalana es parte de una tendencia internacional de reforma del sector público -i.e. New Public Management (NPM). El mayor cambio derivado de estas reformas en numerosos países de la OCDE consiste en pasar de un modelo organizativo jerárquicamente integrado de financiación y provisión de servicios a un modelo de relaciones contractuales entre estructuras desagregadas - casi-mercados - El objetivo de este artículo es, en primer lugar, identificar y definir los componentes de NPM, así como posibles variantes dentro del ámbito específico de las reformas sanitarias. En segundo lugar, se trata de analizar hasta qué punto el proceso de reforma de la Administración sanitaria catalana presenta tales elementos definitorios de NPM, y en qué medida se aproxima a alguno de los modelos de reforma sanitaria identificados.

\subsection{New Public Management: componentes y secuencia}

Según Dunleavy y Hood (1994:9) NPM implica un giro en las dos coordenadas básicas del diseño organizativo del sector público: el control de la discrecionalidad ('grid dimension'), y la diferenciación del sector ('group dimension'). En primer lugar, se está reduciendo el grado en que las normas procedimentales limitan la discrecionalidad de actuación ('down-grid movement').
En segundo lugar, se están atenuando la separación y la diferenciación del sector público frente al sector privado ('downgroup movement).

La presión sobre cada una de estas dimensiones tiene su origen en diferentes componentes de NPM. Así, el giro sobre la primera de ellas deriva de un mayor poder de decisión y actuación para los gestores, de la proliferación de estándares y medidas de actividad explícitas, y del control basado crecientemente en resultados (outputs). Al mismo tiempo, se presiona sobre la segunda coordenada a través de la desagregación de estructuras hasta ahora integradas, la competencia entre unidades autónomas públicas y privadas, y con la adopción de eestilo. de gestión propio del sector privado (Hood, 1994:14).

\begin{tabular}{|l|l|}
\hline \multicolumn{2}{|c|}{ Figura 1 } \\
Componentes de NPM \\
\hline $\begin{array}{c}\text { Menor control de la } \\
\text { discrecionalidad }\end{array}$ & $\begin{array}{c}\text { Menor diferenciación del } \\
\text { sector público }\end{array}$ \\
\hline $\begin{array}{l}\text { 1. Discrecionalidad para } \\
\text { los gestores. }\end{array}$ & 1. Desagregación. \\
$\begin{array}{l}\text { 2. Estándares explícitos y } \\
\text { medidas de actividad. }\end{array}$ & 2. Competencia. \\
3. Control de outputs. & 3. Estilo de gestión del \\
sector privado.
\end{tabular}

Fuente: Adaptado de HoOD (1994:130). 
Estas coordenadas podrían corresponder a la distinción que Polut (1993:188-189) apunta entre las reformas administrativas neo-tayloristas de los ochenta y el NPM de los noventa, respectivamente. El neo-taylorismo, con su énfasis en el control y supervisión exhaustivos, la reducción de costes, la eficiencia, la economía, los indicadores de actividad y remuneración en función de resultados, se habría concentrado en la primera dimensión -es decir, en el componente de NPM que DunLEAVY (1994) define como 'incentivación'. A su vez, el NPM de los noventa, con su énfasis en la calidad, la eficacia, y la orientación al consumidor, se habría centrado en la segunda dimensión. El motivo no habría sido la realización de estos valores en sí, sino las profundas reformas estructurales que los han acompañado y que han contribuido a borrar la separación entre público y privado - -esto es, lo que Dunleavy conceptualiza como los componentes de 'desagregación' y 'competencia' en el NPM.

Partiendo de estos tres conceptos centrales definidos por DUNLEAVY (1994:38-43), podríamos argumentar que las reformas de NPM se basan en la desagregación de estructuras organizativas previamente integradas para impulsar la competencia por la provisión; competencia que, a su vez, se originaria a partir de mecanismos previos de incentivación. La secuencia 'incentivación-desagregación-competencia' implica que la eficiencia-X (eficiencia en la gestión) a través de la incentivación no habría sido conseguida en un principio, lo que habría impul- sado la reestructuración organizativa posterior. Esta desagregación habría sido necesaria para crear una cultura de gestión orientada a la eficiencia. El siguiente paso habría sido la competencia entre unidades desagregadas del sector público y unidades del sector privado por la provisión de bienes y servicios públicos -i.e. competencia por el mercado, y no en el mercado-.

Según Aucoin (1990:235), dos líneas de pensamiento convergen en el desarrollo del NPM. Por un lado, la teoría de public choice, orientada a restablecer la primacía del control político -i.e. del gobierno representativo-, sobre la burocracia, a través de una centralización y supervisión exhaustiva. Por otro lado, la eșcuela 'managerialista', orientada a restablecer la primacía de los principios de la gestión sobre la burocracia, a través de la libertad para gestionar.

En este sentido, podriamos argumentar que la secuencia antes sugerida representa un proceso en el que se reemplaza el control politico a través de incentivos internos por un control económico a través de incentivos externos. Es decir, la reforma de las estructuras organizativas vía desagregación, facilitaría la introducción de mecanismos de competencia, lo que a su vez permitiría obtener los efectos inicialmente esperados de la incentivación. Los incentivos no aumentarían ya los costes del control político, sino que serían generados 'espontáneamente' por la competencia entre proveedores.

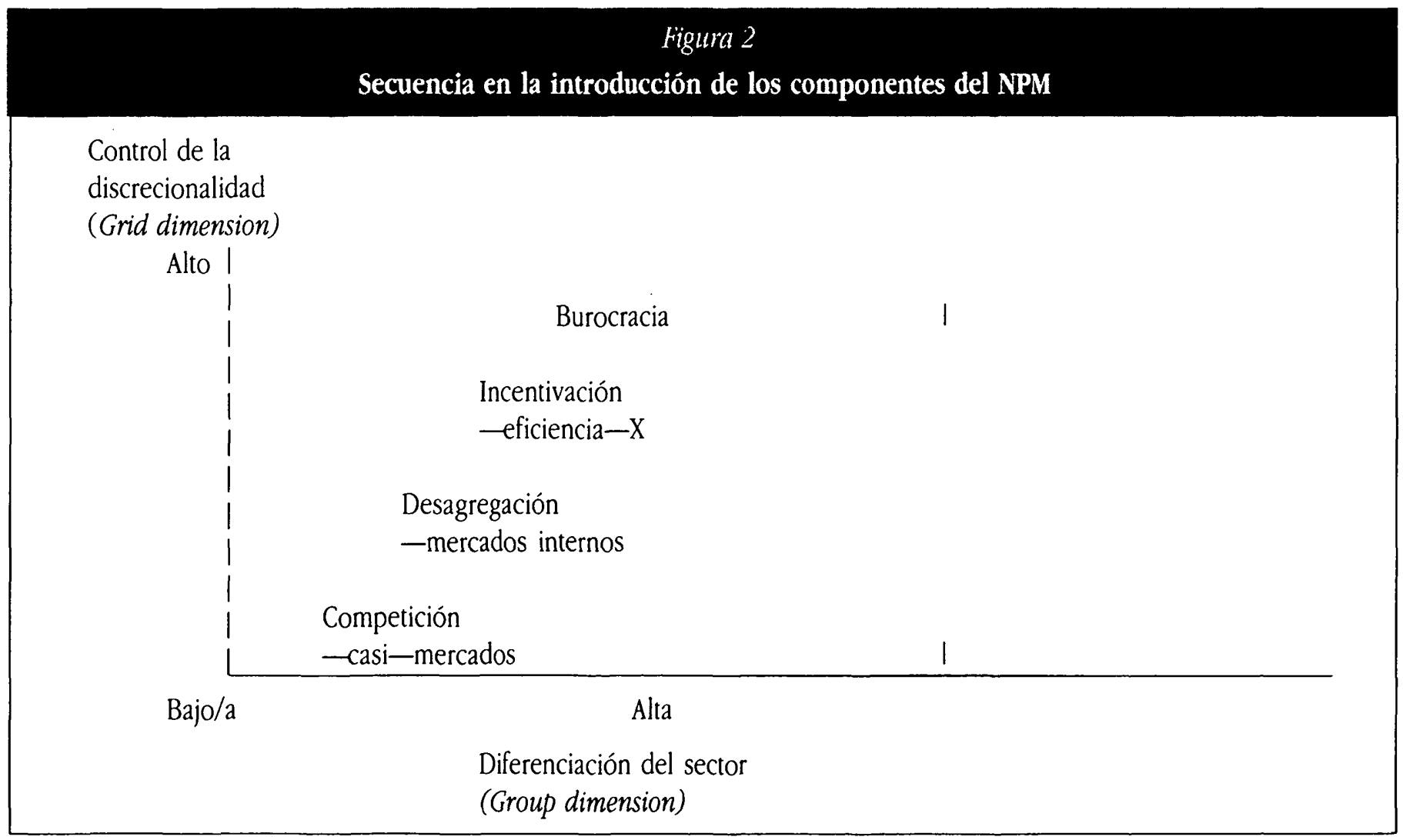

Fuente: Elaboración propia. 
Como muestra la Figura 2, la incentivación habría sido introducida en el sector público mientras su separación y diferenciación estructural respecto al sector privado eran inequivocas. Iniciativas en Gran Bretaña como la Financial Management Initiative (1983) para la función pública, o la Inquiry in the National Health Service Management (primer Informe Griffiths, 1983), ejemplifican esta tendencia.

Sin embargo, pasar de la jerarquía a un sistema de control basado en incentivos sin un cambio en las estructuras organizativas resultó difícil. Así, con la desagregación se pretendió vencer tales obstáculos clarificando responsabilidades políticas y de gestión, y facilitando la devolución de autoridad. Para ello se separaron compradores y proveedores, se desagregaron en unidades pequeñas ejecutivas o proveedoras actividades previamente integradas, y se redisenaron procesos presupuestarios y de personal según prácticas del sector privado. Estas han sido las ideas fuerza de iniciativas británicas como la Next Steps Initiative (1988) para la función pública, y el White Paper «Working for Patients" (1989) para el National Health Service, que han impulsado la creación de mercados internos en el sector público.

La competencia en la función pública británica se introdujo a través de la técnica de Market Testing - dentro del programa Competing for Quality (1991) — . Los 'casi-mercados' no serían sólo internos, es decir, no involucrarían sólo a proveedores públicos sino también privados, compitiendo por contratos que unidades compradoras gestionan. La competencia en el sector sanitario estaba ya contemplada en Working for Patients, autorizando a los General Practinioners a gestionar su propio presupuesto, y a los hospitales a prescindir de la supervisión de las District Health Authorities y convertirse en Trusts independientes.

A diferencia de la función pública, el impacto de NPM en el sector sanitario no se ha circunscrito al ámbito anglosajón -i.e. Gran Bretaña, Nueva Zelanda, Australia y Estados Unidos-, sino que ha afectado a numerosos países de la OCDE. Precisamente, los elementos de convergencia en las reformas sanitarias en marcha se identifican con los componentes de NPM apuntados, y en concreto, con los tres conceptos definidos por DunLEaVy (1994).

\subsection{Reformas de NPM en el sector sanitario}

Según Saltman y Otter (1992) las actuales reformas del sector sanitario se diferencian en dos aspectos clave: las posibilidades para el mercado y la competencia, y el punto de apoyo demanda u oferta-. Los extremos teóricos de este continuum serían los modelos de competencia pública y el de mercado mixto, el primero asemejándose al caso sueco y el segundo al caso británico.
Siguiendo a Saltman y OTter (1992:83-93), en el modelo de competencia puiblica la titularidad y gestión de los proveedores de servicios sanitarios son públicas, como también es público el sistema asegurador - comprador público de servicios. El paciente puede elegir centro y médico, y las asignaciones presupuestarias a los proveedores sanitarios, tanto para inversiones como para incentivos económicos como complemento salarial, se calculan en función de su cuota de mercado (número de pacientes) y de su productividad (eficiencia interna). En definitiva, se trata de un mercado interno al sector público.

En cambio, en el modelo de mercado mixto se asigna un presupuesto a una autoridad pública que ostenta el rol de asegurador/comprador de servicios sanitarios para cubrir las necesidades de la población de su área geográfica. El margen de maniobra de este comprador incluye: la negociación y contratación de provisión pública y privada de servicios sanitarios, sopesando precio y calidad; la venta y transferencia de activos; y la obtención de recursos financieros a través del mercado de capitales.

La diferencia básica entre estos dos modelos es el punto de apoyo desde el que se impulsa la competencia: en el modelo de competencia pública ese rol se asigna al paciente, mientras que en el de mercado mixto corresponde al gerente responsable de los servicios sanitarios del área en cuestión. Es decir, el primer modelo de reforma gira en torno a la demanda, y el segundo en torno a la oferta. En ambos modelos las opciones de voz y salida ofrecen posibilidades diversas y más complejas (LÓPEZ i CASASNOVAS, 1995:144-145).

En estos dos modelos, la desagregación afecta al lado de la provisión. Sin embargo, la fragmentación del poder monopsonístico del asegurador $y$, por tanto, la introducción de competencia en el lado del comprador se identifica con un modelo diferente. En este sentido, SERRA (1994:253) sugiere que la propuesta de la administración Clinton de reformar el sistema de seguro de enfermedad se identificaría con el modelo de competencia gestionada definido por ENTHOVEN. Empresas y organizaciones públicas o casi-públicas actúan como sponsors al contratar cobertura sanitaria obligatoria -determinada por las autoridades públicas-, con compañías aseguradoras privadas (Health Maintenance Organizations). Estas comprarían servicios sanitarios a proveedores públicos y privados. Estos modelos se resumen en la Figura 3.

Los componentes de desagregación y competencia en la organización y funcionamiento de los servicios sanitarios identificados hasta ahora han sido acompañados por la introducción de incentivos. En este sentido, ABEL-Smith (1995:124) subraya algunas movimientos contradictorios. En algunos casos; incentivos para una mayor productividad se introducen mientras sistemas de 'orden y control' (comand-and-control) de organizaciones de servicios se convierten en entramados de contratos 


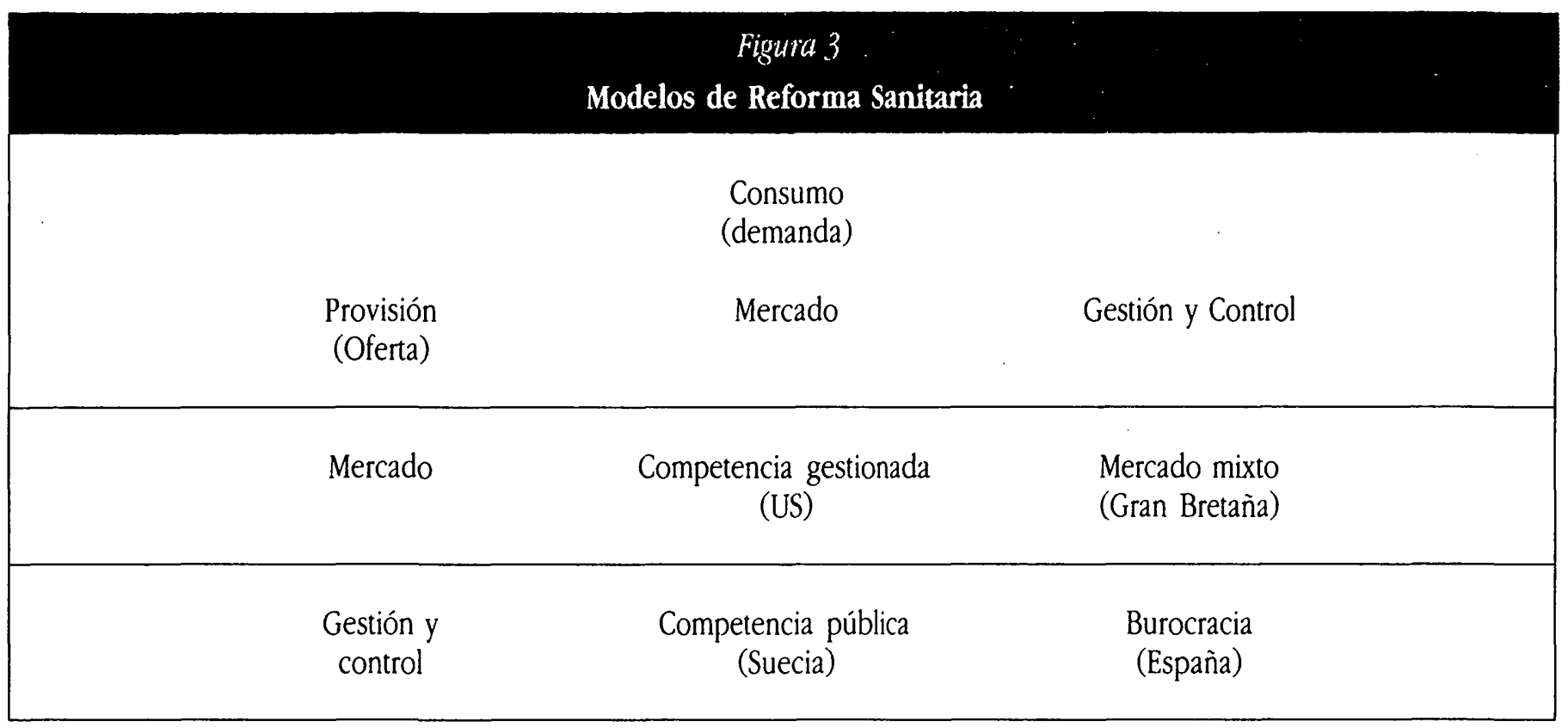

Fuente: Adaptado de SerRa (1994:254).

y sistemas complejos de gestión de servicios. En otros casos, se ha intentado restringir la productividad con medidas como la limitación de la remuneración de los médicos, cambios en las escalas de valor relativo, substitución de presupuesto por pago por día en los hospitales.

Según Williamson (1991:281), la intensidad de los incentivos como instrumento aplicable dentro de estructuras de gobierno alternativas, esto es, modelos organizacionales, está inversamente relacionada a la intensidad de los controles administrativos. Los primeros serían esenciales en el marco de relaciones contractuales o de mercado, mientras que los segundos serían lugar común en estructuras jerárquicas. Por supuesto, los modelos híbridos son los más frecuentes. La paradoja es que en el mercado de servicios sanitarios, demasiados incentivos para la productividad pueden llevar a malgastar recursos por exceso de provisión de servicios, mientras que incentivos escasos pueden malgastar recursos por lentitud de funcionamiento de los servicios (ABEL-SMITH, 1995:124).

La introducción sistemática de incentivación, desagregación (separación comprador/proveedor), y competencia ha conllevado y conllevará una utilización masiva de contratos. Los argumentos que apuntan a una mayor eficiencia derivada de este proceso se basan en los efectos que en este sentido tendrían la especificación de objetivos generales y específicos, la clarificación de líneas de responsabilidad y rendición de cuentas (accountability), y la eliminación del sesgo hacia el productor en la provisión de servicios (originada por la integración de ambas funciones en la misma organización), situando ahora al ciudadano-paciente-consumidor en el lugar prioritario del proceso político- (RANSON y STEWART, 1994:164-166).
Estas reformas de NPM están afectando también a la Administración sanitaria catalana en una forma cuya relevancia, conexión y caracterización respecto a dicha tendencia internacional no ha sido aún analizada.

\section{Reformas en la Administración sanitaria catalana}

\subsection{Ia Llei d'Ordenació Sanitària de Catalunya (LOSC) de 1990}

La LOSC es ambigua, básicamente como resultado del proceso de negociación previo a su aprobación por el Parlament de Catalunya en Julio de 1990. En la Exposición de Motivos de la Ley se apunta la necesidad de separar la responsabilidad de planificar y asegurar la cobertura sanitaria eficiente y eficaz a partir de los recursos disponibles, de la responsabilidad de gestionar la provisión de los servicios sanitarios. Sin embargo, el objetivo explícito de evitar las rigideces derivadas de la estructura y naturaleza jurídica del Institut Català de la Salut (ICS) se iba a conseguir mediante la creación de un nuevo ente público de carácter institucional, el Servei Català de la Salut (SCS), adscrito al Departament de Sanitat $i$ Seguretat Social (DSSS), que asumiria ambas responsabilidades de nuevo (Entrevistas - Sr. Massaguer ', 12/7/95; Sr. Sintes $\left.{ }^{2}, 25 / 7 / 95\right)$. 
En este sentido, de entre las responsabilidades del SCS cabe destacar: (a) la gestión y administración de infraestructuras y servicios del sistema público de salud, ya sea a través de fórmulas de gestión propias de Derecho público o privado; (b) la reorganización, planificación, programación y evaluación de los servicios, así como su financiación -asignación de recursos-. Por lo tanto, el SCS, como ente público de carácter institucional, tiene la responsabilidad de proveer servicios sanitarios, pero tiene libertad para recurrir a fórmulas de gestión tanto públicas como privadas para intervenir en la compleja red sanitaria catalana ${ }^{3}$. Sólo las funciones de regulación y financiación del servicio público de salud están sujetas al Derecho público -administrativo-.

En este sentido, el SCS puede gestionar estos recursos directamente, o indirectamente si así se lo autoriza el Consell Executiu del Govern de la Generalitat. Tal gestión indirecta incluye: la creación de nuevos proveedores -empresas públicas-, el establecimiento de acuerdos para una gestión integrada, la creación de consorcios públicos que a su vez pueden crear organismos de carácter institucional, y la creación o participación en otras organizaciones públicas o privadas (LOSC, art.7).

La descentralización y desconcentración de la gestión son principios centrales de la LOSC (art.2). Así, la estructura organizativa del SCS está compuesta por (1) los Servicios Centrales, encargados de la planificación de recursos, formulación de la política sanitaria y contratación; (2) ocho Regiones Sanitarias, encargadas de la ejecución y control de las políticas del SCS, de la gestión de recursos a través del establecimiento de contratos, y de la planificación dentro de la propia Región; (3) los Sectores Sanitarios, dentro de las Regiones Sanitarias, que integran y proveen a las Areas Básicas de Salud de infraestructura de gestión, y que están encargados de la gestión de recursos y evaluación, debiendo también participar en el proceso de planificación en su ámbito geográfico (DSSS, 1992a:13). En las Áreas Básicas de Salud, los gerentes de los centros sanitarios son responsables de la implementación de los contratos y acuerdos firmados. Esta estructura es la base de una planificación descentralizada, que sigue un proceso ascendente de niveles organizativos inferiores a superiores (bottom-up) para la elaboración del Pla de Salut de Catalunya (SCS, 1992; SCS, 1994).

Los centros sanitarios son instados por la LOSC a desarrollar mecanismos de gestión por objetivos y de control de resultados (outputs), para informar al SCS sobre la valoración económica de sus actividades en base a indicadores sanitarios y económicos (LOSC, art.50). Estos datos han de ser incluidos en el presupuesto de cada centro, que es la base para elaborar el presupuesto de la Región Sanitaria. Este proceso presupuestario descentralizado proporciona a los Gerentes de Región Sanitaria información útil para tomar decisiones tanto respecto a los contratos y acuerdos a diseñar, como a las reasignaciones de recursos a realizar entre proveedores de su Región una vez el ejercicio presupuestario ha empezado. El Director del SCS es quien decide las transferencias de créditos entre centros pertenecientes a diferentes Regiones. En este modelo, los Gerentes de Región Sanitaria no han de interferir en la gestión de los centros sanitarios, sino que han de proporcionarles criterios y argumentos efectivos y eficientes para ayudar, a un nivel estratégico, a los gerentes de los centros sanitarios (BAREA, 1990:27-8).

Hasta aqui, una interpretación 'literal' de la LOSC. En resumen, la intención era que el SCS, como una organización nueva, asumiese las funciones del ICS, el cual dejaría de ser una organización gestora de los servicios sanitarios de la Seguridad Social para integrarse en el SCS. Sin embargo, esta interpretación literal del texto de la LOSC prevalecería únicamente hasta finales de 1990. Para cuando el SCS fue creado en 1991, la política de reforma que de hecho se estaba implementando ya seguía una interpretación bastante diferente. De hecho, la separación entre los roles de comprador y proveedor iba a prevalecer, y el ICS no iba a 'desaparecer' sino a iniciar un profundo proceso interno de refor. ma para convertirse en un gestor y proveedor competitivo de servicios sanitarios públicos (Entrevista $-\mathrm{Sr}$. Massaguer, 12/7/95).

\subsection{El informe McKinsey: La reinterpretación de la LOSC}

Un informe elaborado por la consultora McKinsey \& Company en 1991 a petición del DSSS iba a ser la base para reinterpretar el nuevo modelo. Básicamente, sus recomendaciones subrayaban la necesidad de clarificar responsabilidades mediante la separación de.los roles de comprador y proveedor, así como de habilitar la introducción de un estilo de gestión propio del sector privado en ambas funciones. Se afirmaba que la competencia gestionada facilitaría no sólo la consecución de los objetivos de eficiencia y eficacia, sino también los de unos servicios de calidad orientados al consumidor que la propia LOSC especifica (DSSS, 1992a:4-5).

Se explicita, pues, que el SCS debe adoptar el rol de comprador. Las responsabilidades que ello requiere incluyen definir y evaluar las necesidades sanitarias, es decir, planificar y asegurar su cobertura mediante la asignación de recursos vía contratos con proveedores. Respecto a la planificación, la LOSC ya contempla un proceso de desarrollo ascendente. (bottom$u p$ ), en el que los Sectores Sanitarios, dentro de las Regiones, jugarían un rol clave. La función aseguradora requiere que el SCS establezca objetivos e indicadores de resultados para supervisar el cumplimiento de los contratos y evaluar la eficiencia y calidad de los servicios provistos. Así pues, el SCS no sería responsable de la ineficiencia de los proveedores sino de los 
fallos en la planificación y gestión de la competencia a través de incentivos contractuales (DSSS, 1992a:4-8).

En este modelo, los proveedores deben competir por los contratos del SCS en base a la eficiencia y calidad de sus servicios. El ICS habría de iniciar un proceso de reforma para convertirse en proveedor público de servicios contratado por el SCS, como cualquier otro proveedor, en lugar de recibir una asignación presupuestaria. Siempre según el Informe, la diversificación de proveedores debería ser impulsada también en la asistencia primaria, rompiendo así el monopolio que en este ámbito tiene el ICS. En general, los proveedores deberían adoptar un estilo más empresarial y competitivo desarrollando nuevos sistemas de información y gestión y transformando su cultura organizativa (DSSS, 1992b:9-14).

En definitiva, se busca que la clarificación de responsabilidades conlleve una mayor transparencia de flujos económicos, costes de servicios y eficiencia en la actuación de los proveedores y del conjunto del sistema. Como muestra la Figura 4, este modelo podría en principio encajar con las relaciones institucionales ya existentes.

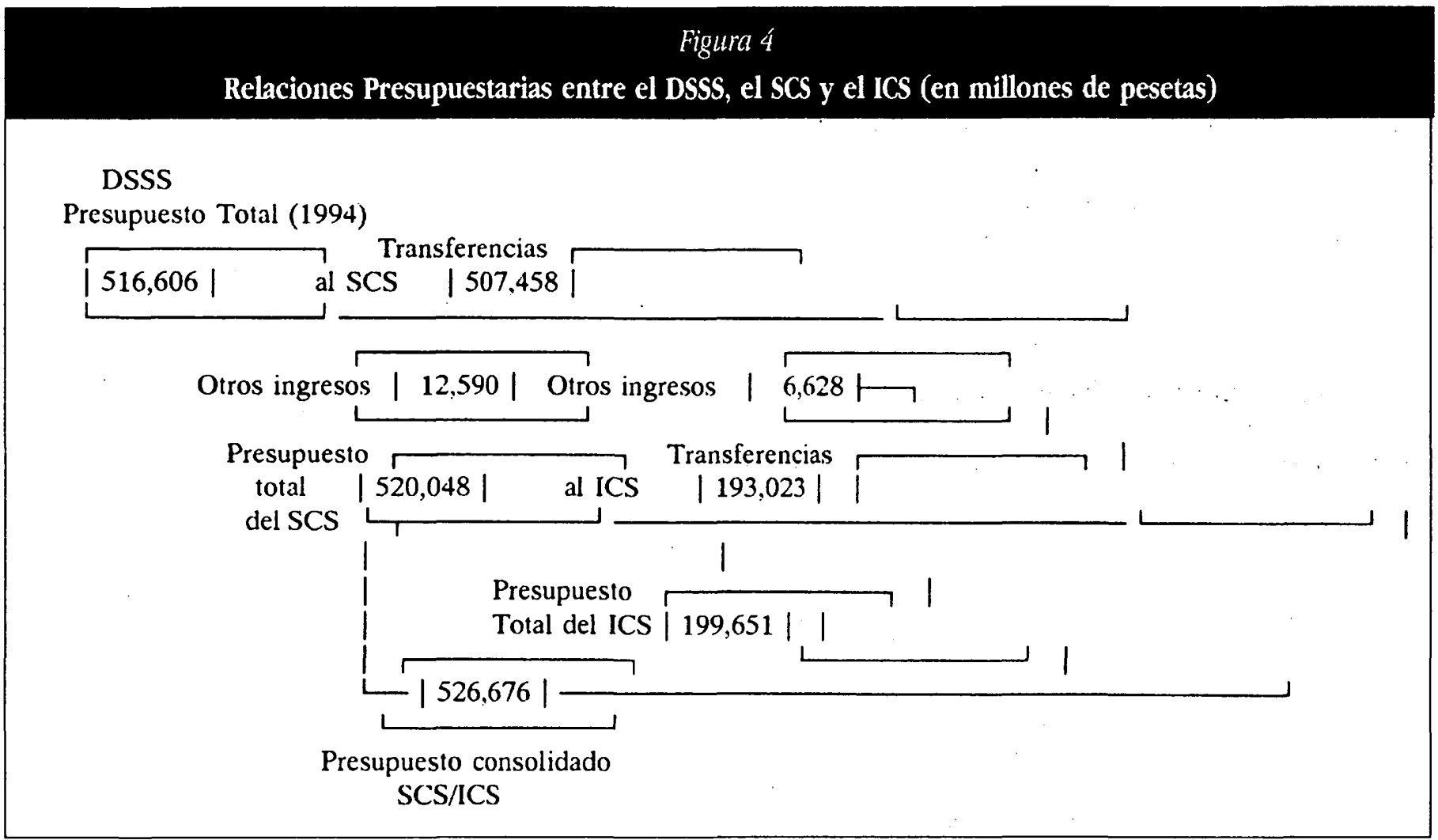

Fuente: Adaptado de DSSS (1994:48).

El presupuesto del DSSS representa la participación de Cataluña en el presupuesto del INSALUD y absorbe casi una tercera parte del presupuesto del Govern de la Generalitat. El DSSS transfiere el $98,23 \%$ de su presupuesto al SCS para la provisión de servicios sanitarios. A su vez, el SCS transfiere el 37\% de su presupuesto total al ICS, y destina el $63 \%$ restante a contratos y conciertos con proveedores privados (DSSS, 1994:94). Existe, pues, un conflicto potencial entre las competencias del SCS para planificar y asignar recursos a las Regiones Sanitarias y dentro de ellas - a través de los Gerentes de Region-, y las competencias del ICS para distribuir su presupuesto entre los proveedores públicos bajo su autoridad, independientemente de su localización geográfica (DSSS, 1994:84). De 1994 en adelante, las Regiones Sanita- rias debían asumir progresivamente las responsabilidades de la asignación de los recursos transferidos del SCS a los centros del ICS dentro de su territorio - un proceso aún en marcha.

\subsection{De las recomendaciones a la práctica: La reforma del ICS}

La diversidad de fórmulas de gestión contempladas en la LOSC ha permitido la puesta en práctica de algunas de las recomendaciones anteriores. El SCS ha ido adoptando un rol de asegurador/ comprador de servicios sanitarios. A un nivel discursivo se han llevado a cabo esfuerzos para explicar el modelo perseguido de 'competencia gestionada' ${ }^{4}$. En éste, el comprador es corresponsable de la viabilidad de los proveedores para asegu- 
rar su compromiso con sus clientes - aquellos cuyas necesidades han de ser cubiertas (MOREU, 1994:23). El principio subyacente a este compromiso es el de equidad, que está explícitamente contemplado en cada norma del marco legal de referencia.

La idea fuerza de este modelo es la necesidad de que el SCS gestione la demanda ', es decir, que la planificación se base en la evaluación de necesidades en lugar de en la estructura de provisión/oferta de servicios sanitarios disponible (Roma, 1994b:13-14). El principal instrumento de planificación es el Pla de Salut de Catalunya. Desde 1993 los contratos han sido concebidos como un instrumento para operacionalizar las directrices especificadas en dicho Plan (1993-5). La consolidación de la Central de Balanços del SCS como instrumento de análisis económico y financiero, junto con el Conjunt de Dades d'Altes Hospitalàries ayudarían a mejorar la definición de los contratos y de la planificación. Otra forma de gestionar indirectamente el mercado de la provisión, y que ha sido ampliamente utilizada por el SCS, es la creación de proveedores directos -empresas públicas-, como organismos autónomos con un amplio margen de maniobra dentro del Derecho privado.

El ICS se halla aún atravesando un profundo proceso de reforma con el objetivo de convertirse en 'solamente-proveedor' público de servicios sanitarios, responsable de la gestión de los recursos económicos transferidos desde el SCS. Debates internos al propio ICS caracterizan la presente situación como sigue: no hay espíritu de dirección ni de grupo y algunos niveles de gestión se consideran superfluos; la calidad apenas se mide -la calidad técnica en los hospitales parece buena pero los servicios a los usuarios son pobres-; el enfoque presupuestario elude medidas de eficiencia en los costes y eficacia; los hospitales del ICS están en desventaja en cuanto a costes frente a sus competidores privados; no existe una 'cultura de la provisión'; no hay una gestión activa de carreras vía incentivos (ICS, 1992:6).

Frente a esta situación, el ICS define así sus propios objetivos: 1. Proveer servicios de calidad de forma competitiva y eficiente; 2. Procurar la satisfacción del cliente dentro del marco contractual establecido con el SCS; 3. Potenciar el desarrollo personal y profesional de sus miembros. En este sentido, se subrayan varios principios de gestión: 1 . Independencia de gestión y responsabilidad sobre resultados -relación contractual con el SCS-; 2. Criterios empresariales incorporando estándares de calidad y conceptos de ingresos y costes; 3 . Cultura organizativa orientada al cliente y basada en la meritocracia interna (ICS, 1994:8-9).

Según expone MASSAGUER (1994:56), tres lineas de reforma están llevándose a cabo en el ICS. Su estructura organizativa se basará no en criterios geográficos sino en líneas de servicios bajo el Gerente del ICS, se despliegan las divisiones del Centro Corporativo, Hospitales, y Asistencia Primaria. Un cambio en el estilo de gestión pretende potenciar la participación de los profesionales y su responsabilidad. Finalmente, se están introduciendo nuevos sistemas e instrumentos de gestión, como auditoría externa, contabilidad y controles financieros, y facturación. A lo largo de 1992 y 1993 la asignación presupuestaria anual del ICS fue 'traducida' a términos contractuales por primera vez -en principio, los hospitales pertenecientes al ICS deben facturar para que las asignaciones presupuestarias que el ICS les distribuye puedan estar relacionadas con los costes por actividad (Roma, 1994a:48-9).

\begin{tabular}{|c|c|c|}
\hline Nivel & Elementos comunes & Elementos especificos \\
\hline Centro Corporativo. & $\begin{array}{l}\text { Dirección y organización de unidades } \\
\text { proveedoras. }\end{array}$ & $\begin{array}{l}\text { Soporte operativo y proceso centra- } \\
\text { lizado de ejecución en atención pri- } \\
\text { maria. }\end{array}$ \\
\hline $\begin{array}{l}\text { Unidades proveedoras de asistencia } \\
\text { primaria. }\end{array}$ & Gestión orientada a los resultados & $\begin{array}{l}\text { Enfoque de red de centros de aten- } \\
\text { ción primaria. }\end{array}$ \\
\hline Hospitales. & Equipos gestores a tiempo completo. & $\begin{array}{l}\text { Enfoque individual más coordina- } \\
\text { ción. }\end{array}$ \\
\hline
\end{tabular}


La Figura 5 muestra la combinación de nuevas estructuras, instrumentos y estilos de gestión en el proceso de reforma. El Centro Corporativo negociaría con el SCS la asignación presupuestaria global del ICS -aunque 'traducida' en términos contractuales, y la distribuiría entre sus centros sanitarios (ICS, 1992:36).. La gestión de recursos por los centros del ICS seguiría un esquema descentralizado (flexibilización de políticas de personal, control de resultados). El desarrollo del modelo llevaria a que cada unidad proveedora firmase un contrato con el Centro Corporativo del ICS. A diferencia de los hospitales, la definición de unidades operativas en el ámbito de la atención primaria apunta hacia la simplificación de niveles jerárquicos y la creación de 'equipos de profesionales de atención primaria' (ICS, 1993:24). En ambos casos, el modelo podria llevarse más lejos en el supuesto de que se estableciesen contratos entre cada unidad operativa/proveedora y el propio SCS, en lugar de con el ICS (Entrevista - Sr. Massaguer, 12/7/95).

\subsection{Legalización de la reinterpretación y de la práctica}

Esta reinterpretación de la LOSC ha sido de hecho puesta en práctica en lo que respecta a varios puntos durante estos cuatro años, y se encuentra también en el origen de la Ley de modificación de la LOSC recientemente aprobada por el Parlament de Catalunya (20 de Septiembre de 1995). Algunos de los principales puntos que se introducen son los siguientes. En primer lugar, se hace insistir en la capacidad del SCS para actuar, por regla general, dentro del Derecho privado en sus relaciones externas -dentro del marco establecido por la Ley reguladora de las empresas públicas catalanas (Llei 4/1985). En segundo lugar, los jefes de unidades operativas (equipos de profesionales de los centros sanitarios) serán miembros del Consejo de Dirección del Sector Sanitario al que pertenecen. En tercer lugar, el SCS está capacitado para establecer contratos para la gestión de centros con profesionales sanitarios. Estos son estimulados a crear 'entidades de base asociativa' con tal objeto. Finalmente, la búsqueda de la diversificación de proveedores requiere que las responsabilidades del ICS sobre la gestión de los servicios sanitarios públicos no sea obstaculizada por las responsabilidades supervisoras del SCS.

\section{Interpretación de las reformas de la Administración}

\section{sanitaria catalana}

\subsection{NPM en la Administración sanitaria catalana: com- ponentes y secuencia}

A la luz del marco teórico definido en el primer apartado, el proceso de reforma de la Administración sanitaria catalana mues- tra componentes centrales de NPM, restricciones explícitas en algunas de sus características y una secuencia de desarrollo poco definida.

Ia desagregación ha sido claramente buscada. El objetivo explícito recogido en la LOSC de separar los roles de comprador y proveedor fue oscurecido al principio por la ambigüedad de la propia ley. El SCS iba a reemplazar al ICS y a responsabilizarse de ambas funciones. La diferencia iba a ser la naturaleza 'autónoma' del SCS y su potencial para desarrollar fórmulas de gestión propias del Derecho privado a la hora de asegurar la provisión de servicios sanitarios. La implementación de dichas fórmulas encontraría terreno adobado en el carácter mixto de la red de provisión catalana.

Si bien el rol proveedor del ICS ha sido claramente definido e implementado -con la consiguiente pérdida de poder por parte del ICS-, el rol comprador del SCS no ha sido 'restrictivamente' definido. Sus funciones de supervisión, derivadas de su responsabilidad de asegurar la cobertura de la población y de salvaguardar los objetivos de la política sanitaria definida (policy objectives), ha justificado su intervención en la gestión de los proveedores tanto a través de la definición de contratos y conciertos, como del propio proceso presupuestario (Entrevistas -Sr. Moreu 6 , 4/4/95; Sr. Sintes, 25/7/95). Se subraya que en una situación de crisis económica y restricciones presupuestarias la libertad de gestión de los proveedores es un principio secundario (Entrevista - Sr. Massaguer, 12/7/95). Igualmente, la separación comprador/proveedor seguirá siendo atenuada por la creación por parte del SCS de nuevos proveedores, a menudo altamente competitivos, a modo de empresas públicas sujetas al Derecho privado —organismos autónomos adscritos al SCS 7 Esta forma de intervenir en la provisión sanitaria también facilita el control sobre los proveedores.

La heterogénea red de hospitales de Cataluña ya conlleva de hecho un alto grado de desagregación en el lado de la provisión privada de servicios sanitarios. En cambio, los hospitales públicos carecen de personalidad jurídica propia. Es el ICS, que agrupa doce hospitales y casi la totalidad de los proveedores de atención primaria en Cataluña, quien tiene tal status como un solo proveedor público. Sin embargo, un futuro desarrollo del modelo de 'competencia gestionada' perseguido contemplaría establecer contratos con proveedores individuales. Un primer paso en esta dirección es la mencionada Ley de modificación de la LOSC (20/9/95). Un objetivo explícito es el de romper el monopolio del ICS en la provisión de atención primaria - equipos de profesionales son animados a crear entidades de base asociativa para optar directamente a contratos con el SCS, y se anima igualmente el desarrollo de proveedores privados. Este proceso se acompañaría de la transformación progresiva de las relaciones laborales en el sector sanitario -de un marco estatutario a otro contractual-. 
En un plano especulativo, este proceso requeriria la posterior desagregación del ICS en sus centros productivos o incluso unidades -equipos de profesionales de los propios centros-, un resultado que podría ser alcanzado transformando el ICS en un bolding de empresas públicas. Se argumenta que la desagregación junto con la individualización de contratos fomentarían incentivos más efectivos y comportamientos más eficientes en los costes. La posibilidad de privatizar dichos proveedores como unidades desagregadas aparece obviamente más cercana, y ha sido un paso relativamente directo en diversos ámbitos del sector público afectados por este tipo de reformas en diversos países.

Sin embargo, existe la voluntad decidida de no desagregar el lado del comprador/asegurador, el cual continuará siendo público - sólo en casos específicos y ya existentes se permitirán aseguradores privados (Entrevista - Sr. Massaguer, 12/7/95). El argumento subyacente es la necesidad de asegurar el control político sobre la gestión y los proveedores. Este objetivo se subraya al definir el modelo de 'competencia gestionada' apuntado por el DSSS. En el caso de un mercado mixto competitivo, se argumenta, el poder monopsonístico del SCS sería decisivo para salvaguardar los objetivos de la política definida. Los proveedores públicos dependerían de hecho de los contratos y conciertos firmados con el SCS.

La incentivación se considera un componente vital para la consecución de una provisión eficiente. Sin embargo, su introducción ha sido lenta. Por ejemplo, la remuneración en función de resultados ha sido introducida en los contratos de los gerentes de segunda línea, pero no en los de los Gerentes de Región Sanitaria, en principio por la oposición del Departament d'Economia $i$ Finances (DEF) de la Generalitat. Además, dentro del propio SCS, la descentralización de la capacidad de decisión sobre la gestión desde los Servicios Centrales o Corporativos a los Gerentes de Región Sanitaria es aún una cuestión a debate. A pesar de que los Gerentes de Región Sanitaria gozan de 'libertad para gestionar' su presupuesto global en orden a obtener la mejor relación calidad-precio, no les corresponde, sin embargo, determinar prioridades en cuanto a la ordenación en el tiempo de los procesos financieros - los Gerentes ordenan los pagos pero no en qué orden han de realizarse, responsabilidad que corresponde al DEF. Según el actual Director del SCS, Sr. Massaguer, el grado de 'libertad para gestionar' ha de estar necesariamente condicionado/restringido tanto por condiciones económicas y presupuestarias como por consideraciones de equidad (Entrevista - Sr. Massaguer, 12/7/95).

Esto es coherente con el modelo de 'competencia gestionada' definido por el DSSS. Así, aunque los Gerentes de Región Sanitaria tienen 'libertad para gestionar' sus presupuestos globales para contratos y conciertos, el grado y la naturaleza de la cobertura definida como cuestión de política sanitaria han de ser alcanzados -incluso si ello requiere subvencionar déficits. Los contratos y la obligación de facturar pretenden incentivar a los proveedores a adaptar de forma progresiva su estructura de costes a los ingresos derivados de sus actividades según se define en el contrato, es decir, a la demanda evaluada y formulada por el SCS.

Sin embargo, algunas críticas apuntan al hecho de que los proveedores aún siguen predominantemente la lógica opuesta (Entrevistas -Sr. Moreu, 4/4/95; Sr. Codina $\left.{ }^{8}, 10 / 4 / 95\right)$. La razón es el conocimiento de que en la mayoría de los casos el SCS no puede prescindir de los servicios que ellos proveen. La relación es, pues, de dependencia mutua. Otros argumentos enfatizan las disfunciones derivadas de combinar una financiación por resultados (outputs), con una planificación e instrumentos de política orientados a los impactos buscados (outcomes) (Entrevista - Sr. Codina, 10/4/95). Este es el caso del actual sistema de contratos y conciertos definidos en términos de resultados, y de las directrices, objetivos y medidas incluidas en el Pla de Salut de Catalunya.

Así, a pesar de que el presupuesto asignado al ICS ha sido 'traducido' a términos contractuales y la facturación es ahora obligatoria, no hay penalización si las condiciones no se cumplen. De hecho, el ICS asigna presupuestos por inputs a sus centros, mientras que los contratos entre el SCS y proveedores privados se redactan en función de los outputs (Entrevista -Sr. Sintes, 25/7/95). En la práctica, la relación SCS-ICS podría calificarse de gestión por objetivos, más que contractual (Entrevista -Sr. Moreu, 4/4/95). Sin embargo, incluso en el caso del ICS, todo el proceso descrito genera flujos de información útil a la hora de comparar y mejorar objetivos y resultados. Además, introducir prácticas de gestión propias del sector privado en el ICS, que está sujeto a las constricciones del Derecho administrativo, es una tarea difícil. Sólo la transformación de su naturaleza jurídica podría facilitarlo (Entrevistas -Sr. Jovells ${ }^{9}$, 12/4/95; Sr. Moreu, 4/4/95).

La incentivación a nivel individual, en lo que respecta a los profesionales del sector sanitario, es un punto polémico. Los profesionales de la sanidad se muestran a favor de los incentivos económicos vinculados a nómina, junto a la desburocratización de su status laboral -siempre que ello no afecte sus intereses adquiridos/creados- (COMB, 1992:25). Como argumenta BOHIGAS (1991:8-9), para diseñar tal sistema de incentivos sería crucial homogeneizar los salarios y las condiciones de empleo en la XHUP, es decir, entre el personal del ICS y el de los proveedores privados. Además, las diferencias tanto en la eficiencia en los costes como en las estructuras de gestión entre los centros del ICS y los proveedores privados deberían también ser atenuadas.

Estas condiciones son también esenciales para que un mercado sea competitivo. La competencia, sin embargo, no ha 
sido reivindicada como rasgo definitorio del modelo de 'mercado gestionado' que se persigue. Se ha argumentado que la eficiencia en la gestión es alcanzable no sólo a través de la competencia, sino también a través de un sistema de contratos basado en los incentivos adecuados (Entrevista - Sr. Moreu, 4/4/95). Un mercado competitivo no se ve como una opción viable debido a los fallos estructurales del mercado inherentes al sector de los servicios sanitarios. En el escenario ideal debería alcanzarse un equilibrio entre la posición monopsonística del SCS y el reconocido poder oligopolístico de muchos de los proveedores.

En resumen, las reformas de NPM en la Administración sanitaria catalana se han caracterizado por un claro movimiento 'down-group'. Tal objetivo fue siempre explícito por parte de la Generalitat - primero favoreciendo la heterogeneidad ya existente en la red de servicios sanitarios catalana; luego, defendiendo la necesidad de homogeneizar la eficiencia en los costes, las estructuras de gestión y las condiciones de empleo en la XHUP para facilitar el diseño de un sistema de incentivos adecuado.

El movimiento 'down-grid' —según lo definíamos en el marco teórico- que también caracteriza las reformas de NPM ha sido obstaculizado por varios factores. En el proceso de reforma están presentes tensiones entre la necesidad de descentralizar poder de decisión en favor de los Gerentes, por un lado, y la voluntad de mantener control político sobre la implementación de la política formulada para asegurar objetivos estratégicos, por otro lado. También, la dificultad de diseñar indicadores y medidas de resultados con efecto en nómina, así como un sistema de incentivos para contratos basados en outputs que sean igualmente vinculantes para todos los proveedores, ha ralentizado el movimiento 'down-grid'.

En conclusión, la secuencia seguida en la introducción de los componentes de NPM en la Administración sanitaria catalana no coincide con la que, según sugeríamos en el marco teórico, se ha registrado en países pioneros en este tipo de reformas. En el caso catalán, la desagregación ha sido introducida como una manera de impulsar un sistema efectivo de contratos en base a incentivos. Sin embargo, no se puede identificar una fase previa que estuviese orientada a conseguir una gestión eficiente a través de medidas neo-tayloristas. De hecho, este proceso de reforma recoge varios componentes en un modelo en que se influyen y refuerzan mutuamente. La competencia, como mera posibilidad, se caracteriza como 'gestionada' por un comprador monopsonístico que controla un sistema de incentivos efectivo.

\subsection{Variantes de NPM en las reformas de los sistemas sanitarios: definiendo el modelo catalán}

El proceso de reforma de la Administración sanitaria catalana expuesto en el apartado 2 incluye variables clave en la dife- renciación de modelos de reforma existentes, según los definíamos en el marco teórico.

En el modelo catalán el margen para el mercado y la competencia se restringe al ámbito de la provisión. Se subraya la importancia del poder monopsonístico de un comprador público y del objetivo de cobertura universal, como características definitorias. En este sentido, el modelo catalán difiere claramente del modelo estadounidense de 'competencia gestionada'. Es más, la coexistencia en el sistema de financiación de la sanidad de impuestos y contribuciones obligatorias a un asegurador público, acerca Cataluña a los modelos sueco de competencia pública y británico de mercado mixto.

A diferencia del modelo sueco de competencia pública, la propiedad y la gestión de los proveedores hospitalarios en Cataluna no son mayoritariamente públicas, y las asignaciones de recursos vía presupuestos tampoco se hacen directamente a los centros proveedores en función de su cuota de mercado. En lugar de ello, al igual que en el modelo británico de mercado mixto, se asigna un presupuesto a una autoridad compradora que es responsable de la cobertura de las necesidades sanitarias de su Región, a través del establecimiento de contratos y conciertos con proveedores privados y públicos.

Por lo tanto, el punto de apoyo para la reforma en el modelo catalán se halla en el lado del comprador. Esto se hace explícito en el rol otorgado al SCS en el 'diseño' del margen para la competencia y para la libertad de gestión en manos de los Gerentes de Región Sanitaria y de los proveedores. Se busca asegurar el control político a través de un sistema de incentivos definido - ya que el mercado de servicios sanitarios es notoriamente imperfecto. Sin embargo, a pesar de que la reforma es intencionadamente conducida desde el lado de la oferta (comprador y proveedores), la planificación de la misma se define como derivada de y orientada por la demanda. El objetivo es adaptar progresivamente la estructura de la provisión a la demanda interpretada por el SCS y expresada posteriormente en la definición de contratos y conciertos. Los proveedores han de competir por los contratos del SCS en lugar de por las preferencias de los pacientes. Actualmente, los contratos aún subvencionan en un grado considerable las necesidades productivas de los proveedores.

Así pues, el modelo catalán tiene varios elementos en común con el modelo británico de mercado mixto. Aun así, las diferencias son también destacables. En el caso británico la desagregación ha tenido efectos más profundos. El poder comprador ha sido fragmentado entre las District Health Authorities (DHA) y los General Practitioners (GP). El poder de negociación de los primeros se ha visto considerablemente reducido. En primer lugar, los criterios de elegibilidad a cumplir por los GPs para gestionar presupuesto propio permite solicitarlo al $50 \%$ de los existentes, sin olvidar que cada uno de estos presu- 
puestos se resta del presupuesto de la DHA correspondiente. En segundo lugar, el monopolio geográfico de los proveedores restringe el margen de maniobra de las DHAs para hacer una elección eficiente de proveedores. A ello hay que añadir que los incentivos perversos derivados del sesgo hacia el productor no han sido eliminados en el caso de los GPs ya que éstos, a diferencia de las DHAs, tienen tanto funciones de compra como de provisión (RANADE, 1994:69-70).

La Ley del pasado 20 de Septiembre de modificación de la LOSC es el primer paso para la desagregación de la provisión sanitaria pública catalana. Profundizar en esta línea llevaría a la desagregación del ICS en sus unidades proveedoras -centros sanitarios-, las cuales pasarían a tener personalidad jurídica propia. En el caso británico, la desagregación fue profundizada en el lado del proveedor. A principios de los 90, las autoridades sanitarias crearon organizaciones compradoras que acordaron amplios 'contratos-presupuesto' con sus propias unidades proveedoras - gestionadas directamente por ellas-. Desde entonces, las unidades proveedoras han sido estimuladas a convertirse en Trusts del National Health Service, desvinculándose de la dependencia (opting out) de las autoridades sanitarias, y siguiendo el argumento de la necesidad de reducir el sesgo hacia el productor a través de la separación de los roles de compra y provisión de servicios (OVRETVEIT, 1995:46). Ciertamente, este proceso presenta claras similitudes con el proceso de reforma que el SCS y el ICS están siguiendo en Cataluña -especialmente, con el proceso de 'pseudoopt-out' definido en la ley antes mencionada.

Las disposiciones centrales del White Paper "Working for Patients apuntaban a la estimulación de la competencia para conseguir eficiencia en los costes. Compradores y proveedores debían competir, los primeros por la best practice, los segundos por el menor precio en orden a atraer negocio. Libertad de gestión y localismo fueron también impulsados vía descentralización del poder de decisión. Se reforzaron los intereses gerenciales pero al mismo tiempo se creó un déficit democrático al abolir los derechos de designación de las autoridades locales sobre los miembros de la ejecutiva de las autoridades sanitarias (MOHAN, 1995:67-72).

En cambio, en el proceso de reforma catalán, la competencia no aparece en publicaciones oficiales como un objetivo prioritario. Como se precisa más arriba, la eficiencia pretende conseguirse a través de un sistema de contratos diseñados en base a incentivos por parte del SCS como autoridad sanitaria. La competencia es de hecho considerada como una característica concebible en fases avanzadas del modeló, aunque siempre subordinada a la primacía de los requisitos de equidad.

Así pues, el modelo catalán que los entrevistados llaman 'competencia gestionada' incluye algunos elementos significativos del 'modelo de mercado mixto', pero con un compromiso explícito con la supervivencia de los proveedores. El movimiento subyacente al proceso de reforma descrito lleva claramente hacia la desagregación y la creación de un cuasi-mercado.

\section{A modo de conclusión}

Experiencias pioneras en países anglosajones respecto a la introducción de NPM en diversos ámbitos del sector público ha consistido en reemplazar en bloque unas estructuras existentes por otras. En cambio, la introducción de NPM en la Administración sanitaria catalana ha seguido la llamada tendencia a "huir del Derecho administrativo'. Esto es, se ha buscado una salida a la presente estructura organizativa explotando el potencial del propio Derecho administrativo de hacer incursiones en el Derecho privado. Lo que podrían considerarse soluciones organizacionales ad boc han sido utilizadas con el objetivo de construir una nueva estructura organizativa y una nueva cultura de gestión.

\footnotetext{
- Estudiante de Doctorado en la London School of Economics and Political Science. 'Director del SCS.

2 Gerente de la Región Sanitaria Ciutal de Barcelona.

${ }^{3}$ En 1990, el 29,9\% de camas hospitalarias pertenecian al ICS (provisión pública transferida del INSALUD), mientras que el 70,1\% eran contratadas por el DSSS a proveedores públicos y privados. Sólo el $16 \%$ de los centros contratados o concertados eran públicos (vinculados a la Administración local y provincial), y sólo el 15\% de las camas contratadas eran públicas (Barea, 1991:28).

${ }^{4}$ Como se verá, este término no define un modelo al estilo del estadounidense referido en el marco leórico.
}

\footnotetext{
5 Curiosamente, no se hace una diferenciación explicita entre demandas y necesidades en los documentos o publicaciones oficiales - las necesidades pueden no llegar a expresarse en términos de demanda.

" Gerente de la Región Sanitaria Centre.

7 Por ejemplo: Instilut de Diagnòstic per la Imatge (1990), Energètica d'Instal.lacions Sanitànies, S.A. (1991), Gestio de Serveis Sanitaris (1992), Gestió i Prestació de Serveis de Salut (1992), Institut d'Assistència Sanitària (1992), Sistemes d'Emergències Mèdiques, S.A. (1992) (DSSS, 1994:64-65).

${ }^{8}$ Director de la Fundació Parc Tauli de Sabadell.

${ }^{9}$ Gerente del ICS.
} 
ABEL-SMITH, B. (1995) - Health care reforms in the OECD countries- en Asociación de Economistas de la Salud, Instrumentos para la gestión en Sanidad. XV Jornadas de Economia de la Salud. SG Editores. Barcelona.

Aucoln, P. (1990) •Administrative reform in public management: Paradigms, principles, paradoxes and pendulums, Governance, 3(2):115-137.

BAREA, J. (1991) -Dictamen respecto a los aspectos económico-financieros de la Ley de Ordenación Sanitaria de Catalunya, Fulls Econòmics del Sistema Sanitari, Monografies, 1:5-34.

BoHIGAS, L. (1991) -La política hospitalària entre els anys 1981 i 1990, Fulls Econòmics del Sistema Sanitari, Monografies, 2:5-9.

CabasÉs, J.; Martin, J. (1995) -Contratos de servicios en el sistema sanitario- en Asociación de Economia de la Salud, Instrumentos para la gestión en Sanidad. XV Jornadas de Economia de la Salud. SG Editores. Barcelona.

COL.IEGI DE METGES DE BARCELONA (1992) L'Informe Abril i els metges. COMB. Barcelona.

Departament de Sanitat I Seguretat Social, Generalitat de Catalunya, (1992a) •El Servei Català de la Salut i la nova organització de l'assistència sanitària pública a Catalunya., Fulls Econòmics del Sistema Sanitari, Informes, 1.

Departament de Sanitat i Seguretat Social, Generalitat de Catalunya, (1992b) •la població, el consum de serveis i l'organització sanitària: opcions per a la millora en l'assignació dels recursos, Fulls Econòmics del Sistema Sanitari, Informes, 2.

Departament de Sanitat I Seguretat Social, Generalitat de Catalunya, (1994) Els recursos econòmics del Departament de Sanitat i Seguretal Social 1994. DSSS. Barcelona.

DUNLEAVY, P. (1994) - The globalization of public services production: Can government be 'best in world'?, Public Policy and Administration, 9(2):36-64.

Dunleavy,P.; Hood, C. (1994) .From Old Public Administration to New Public Management, Public Money and Management, 14(3):9-16.

HooD, C. (1994) Explaining economic policy reversals. Open University Press. Buckingham.

Institut CATALÀ DE la SAlut (1992) - Transformar l'ICS en un proveïdor efectiu de serveis sanitaris en el context de la nova organització de la nova organització de la sanitat pública a Catalunya, Informe interno. Barcelona.
Insmtut Català de la Salut (1993) -Bases de disseny de l'Organizzació de la Divisió d'Atenció Primària., Informe interno. Barcelona.

InSTIUT CATALA DE IA SALUT (1994) L'institut Català de la Salut: d'administració a empresa de serveis. Una proposta per al debat. ICS. Barcelona.

LOPEZ I CASASNOVAS, G. (1995) .Público y privado en sanidad. Claves y estrategias de análisis en Asociación de Economia de la Salud, Cambios en la regulación sanitaria. XIV Jornadas de Economia de la Salud. SG Editores. Barcelona.

Massaguer, R. (1994) •L'Institut Català de la Salut en el marc de la XHUP., Fulls Econòmics del Sistema Sanitari, Monografies, 5:54-57.

Mонам, J. (1995) A National Healtb Service? The restructuring of bealth care in Britain since 1979. St. Martin's Press. New York.

MOREU, F. (1994) tha separació entre compradors i proveidors: cultura, cos de doctrina i instruments en l'experiència de la regió sanitària centre del Servei Català de la Salut, Fulls Econòmics del Sistema Sanitari, 22:21-26.

OVReTVEIT, J. (1995) Purchasing for beallb. Open University Press. Buckingham.

PolutT, C. (1993) Managerialism and the public services. Free Press. New York.

RANADE, W. (1994) A future for the NHS? Health care in the 1990s. Longman. London

Ranson, S.; StrWart, J. (1994) Management for the public domain. St. Martin's Press New York.

Roma, J. (1994a) servei Català de la Salut: passat, present i futur, Fulls Econòmics del Sistema Sanitari, Monografies, 5:47-50.

Roma, J. (1994b) sLa sanitat pública a Catalunya com a element de política económica., Fulls Econòmics del Sistema Sanitari, 22:9-16.

Saltman, R.; OtTer C. (1992) Planned markets and public competition. Strategic reform in northern European bealtb systems. Open University Press. Buckingham.

SERra, A. (1994) -Els models d'organització sanitària• en LOPEZ i CASASNOvAS, G. (Dir) Anàlisisi econòmica de la Sanitat. Generalitat de Catalunya, Departament de Sanitat i Seguretat Social. Barcelona.

Servel Català de la Salut (1992) Què és el Pla de Salut?, Pla de Salut, Quadern n²1. Barcelona.

Servei Català de la Salut (1994) Planificació bospitalària: nous papers, nous instruments, Pla de Salut, Quadern n³. Barcelona.

WILLIAMSON, O. (1991) -Comparative economic organization: the analysis of discrete structural alternatives", Administrative Science Quarterly, 36:269-296. 